\title{
Suppression of Brazier Effect in Multilayered Cylinders
}

\author{
Hiroyuki Shima, ${ }^{1}$ Motohiro Sato, ${ }^{2}$ and Sung-Jin Park ${ }^{3}$ \\ ${ }^{1}$ Department of Environmental Sciences \& Interdisciplinary Graduate School of Medicine and Engineering, \\ University of Yamanashi, 4-4-37 Takeda, Kofu, Yamanashi 400-8510, Japan \\ ${ }^{2}$ Division of Engineering and Policy for Sustainable Environment, Faculty of Engineering, Hokkaido University, Kita-13, \\ Nishi-8, Sapporo, Hokkaido 060-8628, Japan \\ ${ }^{3}$ Department of Urban and Environment Engineering, Incheon National University, 12-1 Songdo-dong, Yeonsu-gu, \\ Incheon 406-772, Republic of Korea
}

Correspondence should be addressed to Sung-Jin Park; park8358775@gmail.com

Received 24 December 2013; Accepted 14 March 2014; Published 28 April 2014

Academic Editor: Zaoyang Guo

Copyright (C) 2014 Hiroyuki Shima et al. This is an open access article distributed under the Creative Commons Attribution License, which permits unrestricted use, distribution, and reproduction in any medium, provided the original work is properly cited.

\begin{abstract}
When a straight hollow tube having circular cross-section is bent uniformly into an arc, the cross-section tends to ovalize or flatten due to the in-plane stresses induced by bending; this ovalization phenomenon is called the Brazier effect. The present paper is aimed at theoretical formulation of the Brazier effect observed in multilayered cylinders, in which a set of thin hollow cylinders are stacked concentrically about the common axis. The results indicate that mechanical couplings between stacked cylinders are found to yield pronounced suppression of the cross-sectional ovalization. Numerical computations have been performed to measure the degree of suppression in a quantitative manner and to explore how it is affected by the variations in the bending curvature, the number of stacked cylinders, and the interlayer coupling strength.
\end{abstract}

\section{Introduction}

Concentrically stacked cylinders are ubiquitous in nature and technology. Primary examples in the field of condensed matter physics is a series of multiwalled nanotubes. To date, successfully synthesized was a wide variety of nanotubes made from carbon [1, 2], silver [3], gold [4], silica [5], boron nitride $[6,7], \mathrm{WS}_{2}[8,9]$, and $\mathrm{TiO}_{2}[10]$, most of which show potential utilities as device components owing to unique physicochemical properties and/or large surface area. In a realm of biochemistry, in addition, there are several examples such as self-assembled microtubules in living cells [11] and multilayer lipid tubes [12]. In those biotubes, the mechanical strength and morphological stability are crucial factors for their biological functions [13-15].

From a practical viewpoint, coaxial multicylinder structures give a merit for designing composite materials both in macroscopic and nanoscopic scales. In civil engineering, for example, deep water pipelines [16] and fiber-reinforced polymer tubes [17] are known to be endowed with high mechanical strength by constructing them with multilayered structures. Choice of multilayered structures is also preferred in building thermal energy storage with cylindrical shape [18], wherein a concentric-tube arrangement results in significant improvement in the system performance.

Another important benefit of coaxial multicylinder structures is enhanced mechanical robustness against crosssectional deformation. Compared with a single hollow cylinder, multilayered counterparts afford resistance to crosssectional deformation caused by external load $[19,20]$. The resistance property originates mainly from the presence of internal cylinders enclosed by outer ones; the internal cylinders can push back the inward deflection of outer cylinders, thus preventing the cross-sections from severe deformation. This will hold true for bending-induced deformation in the cross-section of a hollow cylinder, commonly known as the Brazier effect [21]. Coaxial multicylinders are expected to lead feasible suppression of the Brazier effect in the systems, thus being preferred for designing composite materials that should be undeformable against external load. Despite the fundamental interest, little attempt has been made so far 


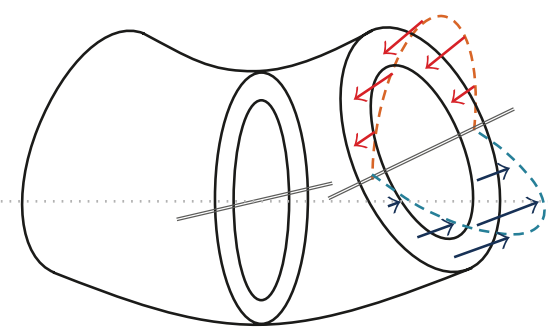

(a)

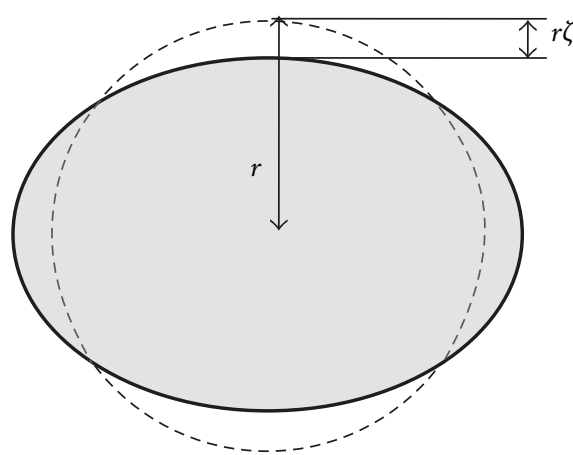

(b)

FIGURE 1: (a) Schematic of the Brazier effect. Bending application to a hollow cylinder of circular section gives rise to the stresses indicated by arrows colored in red and blue. The compressive (red arrow) and tensile (blue) stresses act at a certain angle to the unrotated section, as a result of which the original circular shape deforms into an oval or flattened shape. (b) Definition of the oblateness parameter $\zeta$.

to elucidate the degree of suppression which multilayered structures supply.

The purpose of this work is to establish the theory that quantifies the Brazier effect suppression expected to occur in multilayered cylinders. Thin-shell theory based approach is used to formulate the strain energy of multilayered cylinders under bending, the result of which allows describing the growth of ovalization with increasing the bending curvature. The dependence of the cross-sectional shape on the relevant parameters such as the bending curvature, the number of layers, and the coupling strength between adjacent layers has been also clarified.

\section{Background: What Is the Brazier Effect?}

Flattening of elastic tubes under pure bending has been a focus of much attention over many decades since Brazier first studied this problem [21]. The flattening phenomenon has been usually called the Brazier effect after his name, while other seminal works by Reissner [22] and by Seide and Weingarten [23] have played decisive roles in the progress of the issue.

Figure 1 illustrates the mechanism of Brazier effect. It shows an infinitesimal portion of a deformed hollow cylinder that is originally straight and has a circular cross-section. Application of pure bending to the cylinder causes the compressive and tensile stresses that act at angle to the unrotated section. As a result, the original circular shape deforms into an oval as displayed in the bottom panel of Figure 1. This is equivalent to saying that for a certain curvature, the ovalization minimizes the mechanical energy of the cylinder.

The degree of ovalization is quantified by the parameter $\zeta$, called the oblateness. Given an initially circular cross-section of radius $r$, the product $r \zeta$ equals to the minor axis length of the oval obtained after deformation. The definition of $\zeta$ is conventionally used even for extremely deformed sections, which go beyond simple ovalization to flattening or collapse in a peanut-like shape in which $\zeta$ can be much less than unity.

\section{Formulation}

The stable cross-sectional shape of a hollow cylinder under pure bending is evaluated using a thin shell theory. The theory states that the energy cost $U$ for a cylinder to deform within linear elastic region is written by a sum of three energy terms:

$$
U=U_{\text {cir }}+U_{\text {axi }}+U_{\text {int }}
$$

Here, $U_{\text {cir }}$ is the strain energy associated with the circumferential displacement, $U_{\text {axi }}$ is that with the axial strain, and $U_{\text {int }}$ is the energy caused by the interaction between cylindrical surfaces in the radial direction. All the three energy terms are functions of the curvature $\Gamma$ of the deformed cylinder's axis and the displacement of volume elements. The latter quantity, displacement, is in general represented by a spatially dependent vector. To describe it, we introduce a set of mutually perpendicular vectors $u, v$, and $w$ as shown in Figure 2. Amplitudes of the vectors $u, v$, and $w$ indicate the displacements of a volume element in the radial, circumferential, and axial direction, respectively.

Our objective is a series of the optimal oblateness parameters $\left\{\zeta_{i}\right\}=(i=1, \ldots, N)$ for each $i$ th layer contained in an $N$-layered cylinder; it is what minimizes the total energy $U$ of the system under a given bending curvature $\Gamma$. To this aim, we first derive explicit forms of $U_{\text {cir }}, U_{\text {axi }}$, and $U_{\text {int }}$ for a monolayered cylinder as functions of $u, v$, and $w$ (see from Section 3.1 to Section 3.4), followed by applying the variation method to $U$ with respect to $u$, $v$, and $w$. The strategy for a monolayered system can be extended straightforwardly to multilayered ones, as will be demonstrated in Section 5.

3.1. Stress-Strain Relation. From the definition of elasticity, every elastic medium satisfies an essential relation between applied strain $\left(\varepsilon_{j}\right)$ and the resulting stress $\left(\sigma_{j}\right)[j=r, \theta, z]$, 


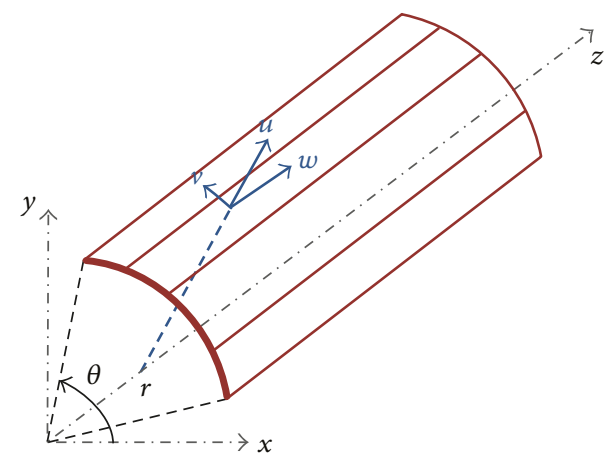

FIGURE 2: Diagram of the displacements $u, v, w$ of a volume element in a hollow cylindrical tube with radius $r$. The tube axis is parallel to the $z$ axis, which is vertical to the tube's cross-section with thickness $h$ that lies in the $x-y$ plane.

which is written in terms of cylindrical coordinates $\{r, \theta, z\}$ by

$$
\begin{aligned}
& \varepsilon_{r}=\frac{1}{E}\left[\sigma_{r}-\nu\left(\sigma_{\theta}+\sigma_{z}\right)\right], \\
& \varepsilon_{\theta}=\frac{1}{E}\left[\sigma_{\theta}-\nu\left(\sigma_{z}+\sigma_{r}\right)\right], \\
& \varepsilon_{z}=\frac{1}{E}\left[\sigma_{z}-\nu\left(\sigma_{r}+\sigma_{\theta}\right)\right],
\end{aligned}
$$

with $E$ being Young's modulus and $\nu$ being Poisson's ratio of the material under discussion. As $\sigma_{r} \equiv 0$ for a hollow cylinder, it follows from (3)-(4) that

$$
\begin{gathered}
\sigma_{\theta}=\frac{E}{1-\nu^{2}}\left(\varepsilon_{\theta}+\nu \varepsilon_{z}\right), \\
\sigma_{z}=\frac{E}{1-\nu^{2}}\left(\varepsilon_{z}+\nu \varepsilon_{\theta}\right) .
\end{gathered}
$$

Suppose that an infinitesimal volume element $d V=d S d z$ of a cylinder is displaced slightly in the $j$-direction $(j=\theta$ or $z$ ). The strain energy $d U_{j}$ caused by the deformation reads

$$
d U_{j}=\frac{1}{2} \sigma_{j} \varepsilon_{j} d S d z . \quad[j=\theta \text { or } z] .
$$

We thus obtain the expressions

$$
\begin{aligned}
& d U_{\mathrm{cir}}=\frac{E}{2\left(1-v^{2}\right)}\left(\varepsilon_{\theta}^{2}+\nu \varepsilon_{\theta} \varepsilon_{z}\right) d S d z, \\
& d U_{\mathrm{axi}}=\frac{E}{2\left(1-v^{2}\right)}\left(\varepsilon_{z}^{2}+\nu \varepsilon_{z} \varepsilon_{\theta}\right) d S d z,
\end{aligned}
$$

where we renamed $d U_{\theta}$ by $d U_{\text {cir }}$ and $d U_{z}$ by $d U_{\text {axi }}$. To proceed the argument, we need to derive explicit functional forms of $\varepsilon_{\theta}, \varepsilon_{z}$ in terms of the displacements $u, v, w$.

3.2. Circumferential Strain Energy. We first consider $U_{\text {cir }}$ of a hollow cylinder subjected to cross-sectional deformation. Suppose a circumferential line element of length $d \widetilde{\ell}_{\theta}$

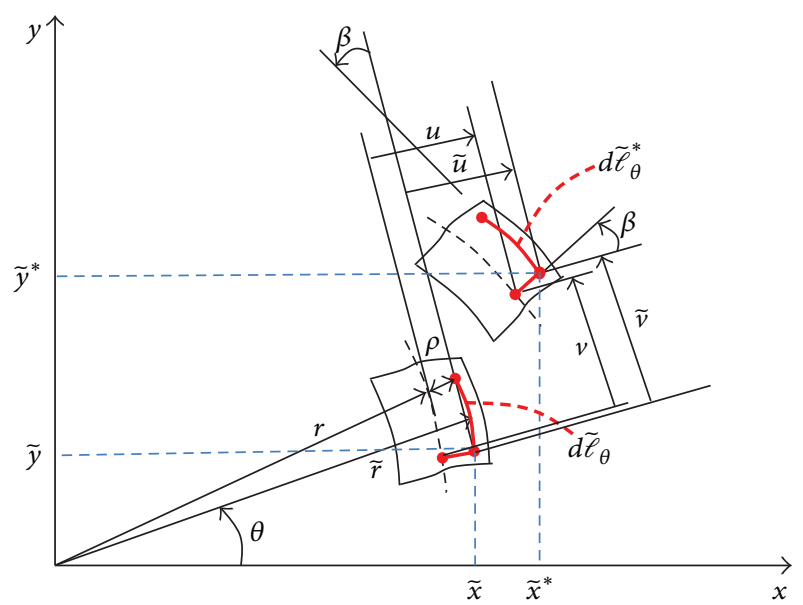

Figure 3: Displacement of a circumferential line element of the length $d \widetilde{\ell}_{\theta}$ to that of $d \widetilde{\ell}_{\theta}^{*}$. The centroidal circle $\mathscr{C}$ of the cross-section, having the radius $r$ before deformation, is also depicted by a dotted curve. Through the displacement described by $\widetilde{u}$ and $\widetilde{v}$, the line element is elongated slightly in the $\theta$ direction and the normal to the centroidal curve rotates with an angle $\beta$.

lying within the annular cross-section with thickness $h$ (see Figure 3$)$. The tilde $(\sim)$ attached to $\ell_{\theta}$ means that it is measured at a point distant from the centroidal circle $\mathscr{C}$. We will see later that under appropriate conditions, the strain is determined by $u(\theta)$ and $v(\theta)$ that are defined just on the undeformed centroidal circle $\mathscr{C}$ of the circular cross-section (see (16)).

The extensional strain of the circumferential line element, designated by $\widetilde{\varepsilon}_{\theta}$, is defined by

$$
\widetilde{\varepsilon}_{\theta}=\frac{d \widetilde{\ell}_{\theta}^{*}-d \widetilde{\ell}_{\theta}}{d \widetilde{\ell}_{\theta}}
$$

where $d \widetilde{\ell}_{\theta}=\widetilde{r} d \theta$ and $d \widetilde{\ell}_{\theta}^{*}$ is the length of the line element after deformation (throughout the paper, the asterisk symbolizes the quantity after deformation). The coordinates $\widetilde{x}^{*}, \tilde{y}^{*}$ of the element after deformation are given by

$$
\begin{aligned}
& \widetilde{x}^{*}(\theta)=[\widetilde{r}+\widetilde{u}(\theta)] \cos \theta-\widetilde{v}(\theta) \sin \theta, \\
& \widetilde{y}^{*}(\theta)=[\widetilde{r}+\widetilde{u}(\theta)] \sin \theta+\widetilde{v}(\theta) \cos \theta,
\end{aligned}
$$

which imply that

$$
\begin{aligned}
\left(d \widetilde{\ell}_{\theta}^{*}\right)^{2} & =\left(d \widetilde{x}^{*}\right)^{2}+\left(d \widetilde{y}^{*}\right)^{2} \\
& =\left[\widetilde{r}^{2}+2 \widetilde{r}(\widetilde{u}+\widetilde{v})+(\widetilde{u}+\widetilde{v})^{2}+(\widetilde{u}-\widetilde{v})^{2}\right](d \theta)^{2} .
\end{aligned}
$$

Note that (8) can be rewritten as $\widetilde{\varepsilon}_{\theta}+1=d \widetilde{\ell}_{\theta}^{*} /(\widetilde{r} d \theta)$. Squaring the both sides and then rearranging give

$$
\widetilde{\varepsilon}_{\theta}+\frac{1}{2} \widetilde{\varepsilon}_{\theta}^{2}=\frac{1}{2}\left[\frac{1}{\widetilde{r}^{2}}\left(\frac{d \widetilde{\ell}_{\theta}^{*}}{d \theta}\right)^{2}-1\right] .
$$




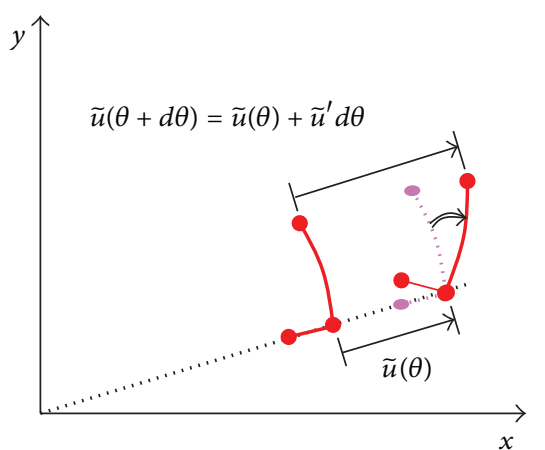

(a)

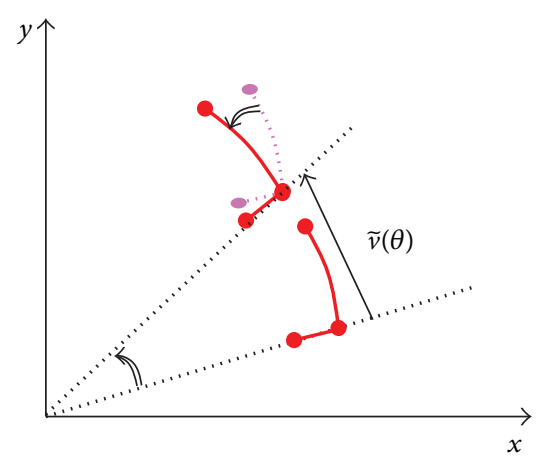

(b)

Figure 4: Two different mechanisms of the rotation of a line element. Left: clockwise rotation due to the spatial variation of $\tilde{u}$ in the $\theta$ direction. Right: counterclockwise rotation caused by the circumferential displacement of the element.

For $\widetilde{\varepsilon}_{\theta} \ll 1$, the term $\widetilde{\varepsilon}_{\theta}^{2}$ can be omitted; we substitute (10) into (11) to obtain

$$
\widetilde{\varepsilon}_{\theta}=\frac{\tilde{u}+\widetilde{v}^{\prime}}{\widetilde{r}}+\frac{1}{2}\left(\frac{\tilde{u}^{\prime}+\widetilde{v}^{\prime}}{\tilde{r}}\right)^{2}+\frac{1}{2}\left(\frac{\tilde{u}^{\prime}-\widetilde{v}}{\widetilde{r}}\right)^{2},
$$

where $\tilde{u}^{\prime} \equiv \partial \tilde{u} / \partial \theta$ and so forth.

The last term in (12) is associated with the rotation of the line element due to deformation. The rotation angle $\beta$ consists of two parts (see Figure 4): (i) a clockwise component $\partial \tilde{u} /(\tilde{r} \partial \theta)$ due to the spatial variation of $\tilde{u}$ in the circumferential direction and (ii) a counterclockwise one $\widetilde{v} / \widetilde{r}$ due to the circumferential displacement of the element. Combination of the two parts gives

$$
\beta=\frac{\widetilde{v}-\tilde{u}^{\prime}}{\tilde{r}},
$$

which has a positive value in the counterclockwise sense.

Formula (12) is valid for arbitrary large rotation $\beta$. Particularly when $\widetilde{\varepsilon}$ and $\beta$ are both sufficiently small (but finite), we may neglect the second term in the right side in (12) (here we exclude the possibility that $|\widetilde{u}|$ or $\left|\widetilde{v}^{\prime}\right|$ is of the order of $\widetilde{r}$ or larger). We further assume that normals to the undeformed centroidal circle $\mathscr{C}$ remain normal and inextensional during the deformation. Under this assumption, we can write

$$
\widetilde{u}=u, \quad \widetilde{v}=v+\rho \beta,
$$

where $u$ and $v$ denote the displacements of a point just on $\mathscr{C}$ and $\rho$ is a radial coordinate measured from $\mathscr{C}$. By substituting (14) into (12), we attain the strain-displacement relation such as

$$
\widetilde{\varepsilon}_{\theta}(\rho, \theta)=\varepsilon_{\theta}(\theta)+\rho \kappa_{\theta}(\theta),
$$

with the definitions

$$
\varepsilon_{\theta}=\frac{u+v^{\prime}}{r}+\frac{1}{2}\left(\frac{u^{\prime}-v}{r}\right)^{2}, \quad \kappa_{\theta}=-\frac{u^{\prime \prime}-v^{\prime}}{r^{2}} .
$$

Here $r$ is the radius of the undeformed circle $\mathscr{C}$.
The results (15) and (16) state that the circumferential strain at points distant from $\mathscr{C}$ is determined by the displacements $u(\theta)$ and $v(\theta)$ of a point just on $\mathscr{C}$. The results also allow us to write the deformation energy $U_{\text {cir }}$ as

$$
U_{\text {cir }}=\frac{E r}{2\left(1-v^{2}\right)} \int_{0}^{L} d z \int_{-h / 2}^{h / 2} d \rho \int_{0}^{2 \pi} d \theta\left[\widetilde{\varepsilon}_{\theta}(\rho, \theta)\right]^{2}
$$

or, more concisely,

$$
U_{\text {cir }}=\frac{E h r}{2\left(1-v^{2}\right)} \int_{0}^{L} d z \int_{0}^{2 \pi} d \theta\left(\varepsilon_{\theta}^{2}+\frac{h^{2}}{12} \kappa_{\theta}^{2}\right),
$$

which implies that $U_{\text {cir }}$ is a functional of $u, v$, and their derivatives as

$$
U_{\text {cir }}=U_{\text {cir }}\left[u, \frac{\partial u}{\partial \theta}, \frac{\partial^{2} u}{\partial \theta^{2}}, v, \frac{\partial v}{\partial \theta}\right]
$$

3.3. Axial Strain Energy. Parallel discussions to that in Section 3.2 give us the explicit form of the deformation energy $U_{\text {axi }}$ caused by the axial strain. The strain $\widetilde{\varepsilon}_{z}$ of the axial line element $d \ell_{z}$ is defined by

$$
\widetilde{\varepsilon}_{z}=\frac{d \widetilde{\ell}_{z}^{*}-d \widetilde{\ell}_{z}}{d \widetilde{\ell}_{z}}
$$

Here $d \widetilde{\ell}_{z}^{*}$ is the length of the line element after deformation. Using the displacement components $\widetilde{u}, \widetilde{v}$, and $\widetilde{w}$, the square of $d \widetilde{\ell}_{z}^{*}$ is given by

$$
\left(d \widetilde{\ell}_{z}^{*}\right)^{2}=\left(d \tilde{\ell}_{z}+\frac{\partial \widetilde{w}}{\partial z} d z\right)^{2}+\left(\frac{\partial \widetilde{u}}{\partial z} d z\right)^{2}+\left(\frac{\partial \widetilde{v}}{\partial z} d z\right)^{2} .
$$

Note the equivalence of $d \widetilde{\ell}_{z}$ with $d z$ in (21); then, we obtain from (20)-(21) that

$$
2 \widetilde{\varepsilon}_{z}+\widetilde{\varepsilon}_{z}^{2}=2 \widetilde{w}^{\prime}+\left(\widetilde{w}^{\prime}\right)^{2}+\left(\widetilde{u}^{\prime}\right)^{2}+\left(\widetilde{v}^{\prime}\right)^{2},
$$

where $\widetilde{w}^{\prime} \equiv \partial \widetilde{w} / \partial z$ and so forth. Since $\widetilde{\varepsilon}_{z}^{2} \ll 1$ and $\left(\widetilde{w}^{\prime}\right)^{2} \simeq 0$, we have

$$
\widetilde{\varepsilon}_{z}=\widetilde{w}^{\prime}+\frac{1}{2}\left(\widetilde{u}^{\prime}\right)^{2}+\frac{1}{2}\left(\widetilde{v}^{\prime}\right)^{2} .
$$


In general, $\widetilde{w}$ differs from $w$ that is allocated just on $\mathscr{C}$. Nevertheless, $\widetilde{w}$ can be related with $w$ and $u$ by

$$
\widetilde{w}=w-\rho \frac{\partial u}{\partial z}
$$

and accordingly $\widetilde{w}^{\prime}=w^{\prime}-\rho u^{\prime \prime}$. Similarly, we use the approximations of $\tilde{u} \simeq u$ and $\widetilde{v} \simeq v+\rho \beta$, which imply that $\widetilde{u}^{\prime} \simeq u^{\prime}, \widetilde{v}^{\prime} \simeq v^{\prime}$. Consequently we have $\widetilde{\varepsilon}_{z}=\varepsilon_{z}-\rho \kappa_{z}$ with the definitions of

$$
\varepsilon_{z}=w^{\prime}+\frac{1}{2}\left(u^{\prime}\right)^{2}+\frac{1}{2}\left(v^{\prime}\right)^{2}, \quad \kappa_{z}=u^{\prime \prime},
$$

which leads us to the axial energy expression

$$
U_{\mathrm{axi}}=\frac{E h r}{2\left(1-v^{2}\right)} \int_{0}^{L} d z \int_{0}^{2 \pi} d \theta\left(\varepsilon_{z}^{2}+\frac{h^{2}}{12} \kappa_{z}^{2}\right) .
$$

It should be emphasized that the expression (26) implies the functional property of $U_{\text {axi }}$ represented by

$$
U_{\mathrm{axi}}=U_{\mathrm{axi}}\left[\frac{\partial u}{\partial z}, \frac{\partial^{2} u}{\partial z^{2}}, \frac{\partial v}{\partial z}, \frac{\partial w}{\partial z}\right] .
$$

3.4. Interaction Energy. Mechanical stability of cross-sections in multilayered cylinders owes in part to interaction between adjacent cylindrical surfaces. In typical nanotubular materials, for instance, the interaction originates from intermolecular van der Waals forces, thus sufficiently small compared with strong chemical bonding [1]; as a result, many nanotubular materials are endowed with anisotropy in mechanical stiffness, characterized by large stiffness against longitudinal stresses and flexibility to radial compression.

For a linear elastic deformation, it is reasonable to represent the interaction energy between $i$ th and $j$ th concentric cylinders by

$$
U_{\mathrm{int}}^{(i, j)}=\frac{k r_{i}}{2} \int_{0}^{L} d z \int_{0}^{2 \pi} d \theta\left(u_{i}-u_{j}\right)^{2}
$$

Here, $k$ is an effective spring constant per surface area $r d \theta d z$, which serves as a measure for the interaction strength. The value of $k$ is dependent on the material and geometric structure of the system considered; among choices, we hereafter pay attention to the weak interaction for which $k r_{N} / E \leq 1$ or lesser, by referring to the existing multiwalled nanotubes [24].

\section{Displacement Condition}

To evaluate the optimal value of $\zeta_{i}$ for each $i$ th cylinder, we need to relate $\zeta_{i}$ with $u_{i}, v_{i}$, and $w_{i}$, through which the total strain energy $U$ is set to be a function of $\zeta_{i}$. In his pioneering work, Brazier hypothesized that a typical crosssection deforms according to [21]

$$
u_{i}=r_{i} \zeta_{\text {inext }} \cos 2 \theta
$$

where the parameter $\zeta_{\text {inext }}$ is a dimensionless measure of the ovalization. The suffix "inext" means that the cross-section is assumed to deform inextensionally in the $\theta$ direction, namely, when $\varepsilon_{\theta}$ in (18) is set to zero. Under this special restriction, one obtains exactly the relation [25]

$$
\frac{\partial v_{i}}{\partial \theta}+u_{i}=0
$$

or equivalently

$$
v_{i}=-\frac{r_{i}}{2} \zeta_{\text {inext }} \sin 2 \theta,
$$

where the constant of integration was set to zero since there is no net rotation of the section about the cylinder axis.

The relation (30) is exact when the circumferential elongation/contraction of volume elements is forbidden $\varepsilon_{\theta} \equiv$ 0 . Even though such the circumferential deformation is permitted, the relation (30) nearly holds in a realm of linear elastic deformation. This is why we use the following expressions for displacement of volume elements contained in the $i$ th cylinder:

$$
\begin{gathered}
u_{i}=r_{i} \zeta_{i} \cos 2 \theta, \quad v_{i}=-\frac{r_{i}}{2} \zeta_{i} \sin 2 \theta, \\
w_{i}=\Gamma\left(z-\frac{L}{2}\right)\left[\left(r_{i}+u_{i}\right) \sin \theta+v_{i} \cos \theta\right],
\end{gathered}
$$

where $\zeta_{i}$ is the oblateness whose value depends on $i$. The validity of the above expressions will be revisited in Appendix.

\section{Optimal Ovalization}

Discussions made in the preceding two sections allow us to formulate the total mechanical energy $U$ as a function of the series of $\left\{\zeta_{i}\right\}$ :

$$
\begin{aligned}
U & =\sum_{i=1}^{N} U_{\mathrm{cir}}^{(i)}+\sum_{i=1}^{N} U_{\mathrm{axi}}^{(i)}+\sum_{i, j(\neq i)}^{N} U_{\mathrm{int}}^{(i, j)} \\
& =\left[\zeta_{\mathrm{i}} \text { 's polynomial of the } 4 \text { th degree }\right] .
\end{aligned}
$$

The optimal ovalization is determined by

$$
\frac{\partial U}{\partial \zeta_{i}}=0 . \quad(i=1, \ldots, N) .
$$

To calculate (35), we can omit $\zeta_{i}$-terms of higher order than the 2 th degree since $\zeta_{i}<1$ for all $i$. The resulting equation with respect to $\zeta_{i}$ can be reduced to the matrix representation of

$$
\mathrm{M} \zeta=\gamma
$$

Here, $\zeta$ and $\gamma$ are column vectors defined by

$$
\zeta=\left[\begin{array}{c}
\zeta_{1} \\
\zeta_{2} \\
\vdots \\
\zeta_{N-1} \\
\zeta_{N}
\end{array}\right], \quad \gamma=\frac{3}{4} \Gamma^{2} E h\left[\begin{array}{c}
r_{1}^{3} \\
r_{2}^{3} \\
\vdots \\
r_{N-1}^{3} \\
r_{N}^{3}
\end{array}\right],
$$


and $\mathbf{M}$ is an $N \times N$ tridiagonal matrix

$$
\mathbf{M}=\left[\begin{array}{cccccccc}
a_{1} & b_{1} & 0 & \cdots & & & \cdots & 0 \\
b_{1} & a_{2} & b_{2} & & & & & \vdots \\
0 & b_{2} & a_{3} & b_{3} & & & & \\
\vdots & & \ddots & & & & & \\
& & & b_{i-1} & a_{i} & b_{i} & & \\
& & & & \ddots & & & \vdots \\
\vdots & & & & & b_{N-2} & a_{N-1} & b_{N-1} \\
0 & \cdots & & & \ldots & 0 & b_{N-1} & a_{N}
\end{array}\right]
$$

with the definitions of

$$
\begin{gathered}
a_{1}=\omega_{1}+\lambda_{1}+k r_{1}^{2} r_{2}, \\
a_{i}=\omega_{i}+\lambda_{i}+k r_{i}^{2} r_{i+1}+k r_{i}^{3}, \quad(2 \leq i \leq N-1) \\
a_{N}=\omega_{N}+\lambda_{N}+k r_{N}^{3}, \\
b_{i}=-k r_{i} r_{i+1}^{2}, \quad(1 \leq i \leq N-1) \\
\omega_{i}=\frac{3 E h^{3}}{4 r_{i}}, \quad \lambda_{i}=\frac{5}{8} \Gamma^{2} E h r_{i}^{3} .
\end{gathered}
$$

As a consequence, the optimal values of $\zeta_{i}$ are determined by

$$
\zeta=\mathbf{M}^{-1} \gamma
$$

from which we derive the dependence of the optimal $\zeta_{i}$ on the curvature $\Gamma$ as formally expressed by $\zeta_{i}=\phi(\Gamma)$.

\section{Numerical Results}

The strategy for evaluating the optimal $\zeta_{i}$ is summarized as follows.

(i) Prepare the energy expressions of $U_{\text {cir }}, U_{\text {axi }}$, and $U_{\text {int }}$ given by (18), (26), and (28), respectively.

(ii) Substitute the displacement expressions of $u_{i}, v_{i}$, and $w_{i}$, given by (32)-(33), into the energy expressions.

(iii) Through (i) and (ii), the total energy $U$ has been reduced to the $\zeta_{i}$ 's polynomial of the 4 th order.

(iv) Omit the 3 rd and 4 th order terms in the $\zeta_{i}$ 's polynomial and make the partial derivatives of $\partial U / \partial \zeta_{i}=0$ to obtain the matrix equation $\mathbf{M} \zeta=\boldsymbol{\gamma}($ see (36)).

(v) Solve (40), $\zeta=\mathbf{M}^{-1} \boldsymbol{\gamma}$, to reach the optimal oblateness $\left\{\zeta_{i}\right\}$.

In actual computations, we consider that all the $N$-layered cylinders with different $N$ possess the same value of $r_{N}$; namely, the radius of the outmost cylinder is fixed to be a common constant $r_{N}$ irrespective of $N$. Taking the common $r_{N}$ as a unit of length, we set the layer's thickness to be $h / r_{N}=$ 0.02 . The remaining parameters to be determined are $N, \Gamma$, and $k$; those values are artificially tuned in order to explore the effect of their variations on the optimal cross-section.
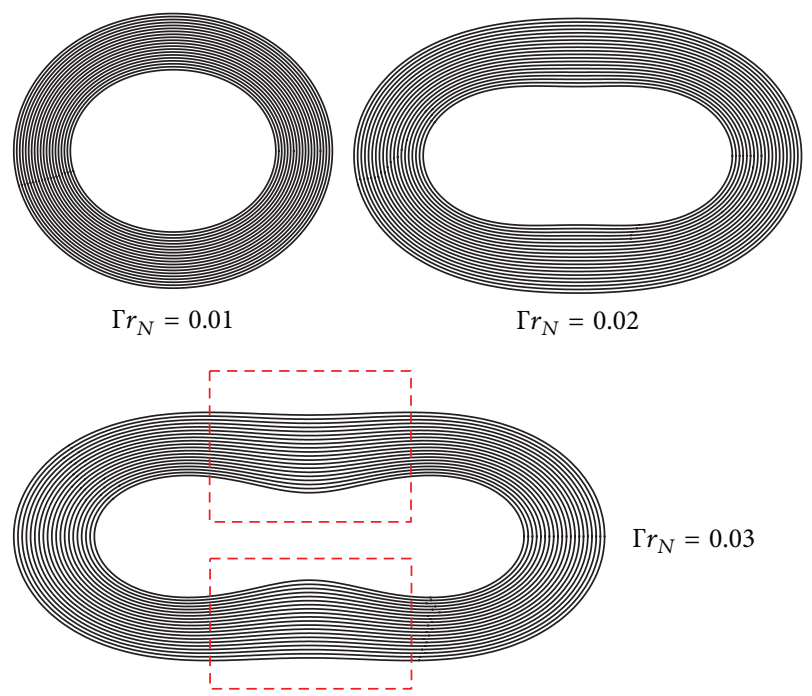

FIGURE 5: Variation in the cross-sectional view with increasing the curvature $\Gamma$ of the cylinder's axis. The parameters of $N=20$ and $k r_{N} / E=0.1$ are fixed.

Figure 5 displays the stable cross-sections under the bending curvature indicated. The curvature is normalized by multiplying the outmost cylinder's radius $r_{N}$; thus, $\Gamma r_{N}$ is dimensionless. For every three samples, we fixed $N=20$ and interlayer coupling strength of $k r_{N} / E=0.1$. It follows from the views that the ovalization gradually evolves with increasing $\Gamma$ as expected. Particularly at $\Gamma r_{N}=0.03$, the outmost layer reaches flattened shape, while the innermost layer nearly collapses into a peanut-like shape. The two bulges indicated by dashed squares imply that large excess strain is stored in the inner layers around the bulgy regions, which prevent from significant deformation of the outer layers by pushing them outward. We thus can say that the physical origin of the Brazier effect suppression in multilayered systems is the present of additional layers embedded into the hollow core of the surrounding layers.

We have found in computations that the degree of suppression monotonically increases with the number of constituent layers $N$. Figure 6 exhibits the dependence of $\zeta_{N}$ on $N$ and $\Gamma$, which tell us that the larger $N$ we apply, the smaller $\zeta_{N}$ we obtain. It is also observed that for $N \geq 40$, the suppression no longer works effectively and the optimal $\zeta_{N}$ converges to a constant.

Added to $N$, the interlayer coupling quantified by $k$ is another important factor for determining the stable crosssection. Figure 7(a) gives examples on how the variation in $k$ contributes to the suppression of the Brazier effect. The horizontal axis indicates the interlayer spring constant normalized by multiplying $r_{N} / E$, by which it is nondimensional. The sample size is fixed to be $N=50$, and the change in $\zeta_{N}$ with increasing $k$ is plotted. The results show that the degree of suppression is magnified with intensifying the couplings up to $k r_{N} / E \sim 0.1$, above which an increase in $k$ gives minor contribution. The pronounced suppression at the small $k$ region is observed universally as far as the sample is composed of modest number of layers, say $N \geq 20$. This 


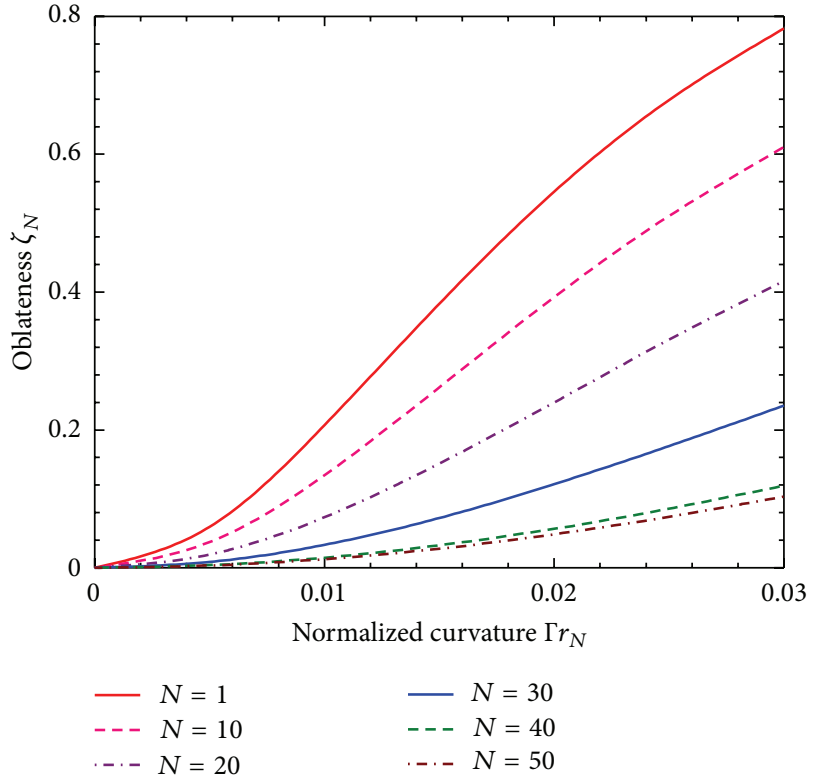

(a)

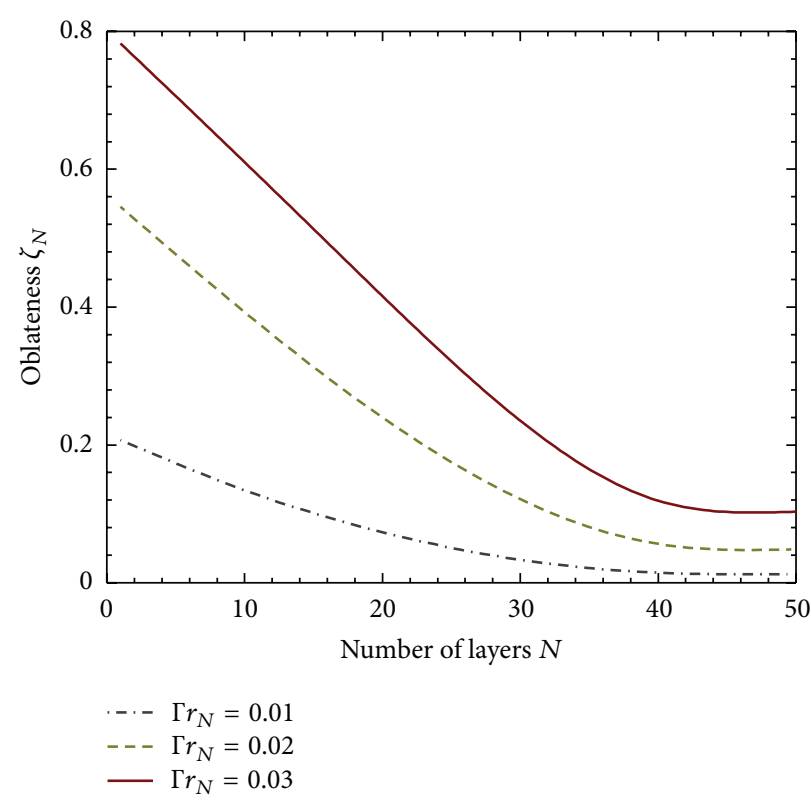

(b)

Figure 6: (a) Oblateness $\zeta_{N}$ of the outmost cylinder in an $N$-layered system as a function of the curvature $\Gamma r_{N}$. The curvature is normalized by the radius of the outmost cylinder $r_{N}$. (b) Suppression in the oblateness $\zeta_{N}$ with increasing the number of constituent layers $N$.

fact is demonstrated graphically in Figure 7(b), which shows the difference, designated by $\delta_{N}$, in the short radius $r_{N} \zeta_{N}$ of deformed cross-sections between the weak and strong coupling conditions defined as follows:

$$
\delta_{N} \equiv\left(r_{N} \zeta_{N}\right)_{k r_{N} / E=0.001}-\left(r_{N} \zeta_{N}\right)_{k r_{N} / E=0.1} .
$$

The plot makes clear that the cross-section endowed with strong interlayer couplings tends to retain its original circular shape when $N \geq 20$. Figure 8 displays, as examples, the robust behaviors of samples with $N=30$ and 50, together with collapse of those with $N=1$ and 10. Inward convex bulges observed in the latter two samples disappear in the former two, which is a manifestation of the coupling effect in modest$N$-layered systems.

\section{Conclusion}

We have proposed an approximation theory that describes the bending-induced ovalization in the circular cross-section of multilayered cylinders. Taking into account the in-plane deformation within individual layers and interlayer couplings, we have formulated the mechanical energy of multilayered cylinders, followed by variational computation to evaluate the optimal oblateness that minimizes the energy under pure bending. The oblateness was found to decrease monotonically with increasing the number of layers and/or strength in the interlayer coupling, as we demonstrated in a systematic and quantitative manner. The results will facilitate the basic understanding of the mechanics of coaxial multicylinder structures that are present universally in macroscopic and nanoscopic science.

\section{Appendix}

\section{On the Validity of (32)}

In this Appendix, we examine the validity of our approximation as restated by

$$
u_{i}=r_{i} \zeta_{i} \cos 2 \theta, \quad v_{i}=-\frac{r_{i}}{2} \zeta_{i} \sin 2 \theta,
$$

which describe the cross-sectional deformation. We should be reminded that the above expressions of $u_{i}$ and $v_{i}$ are natural extensions of the following:

$$
u=r \zeta_{\text {inext }} \cos 2 \theta, \quad v=\frac{\partial u}{\partial \theta},
$$

which are exactly correct under the ideal condition that the circumferential strain (i.e., $\varepsilon_{\theta}$ in (18)) must be zero. Therefore, it is nontrivial if we are allowed to make the straightforward extension to the case, where $\varepsilon_{\theta} \neq 0$, just by replacing the coefficient from $\zeta_{\text {inext }}$ to $\zeta_{i}$ with keeping the function forms of $u_{i}$ and $v_{i}$. We will demonstrate below that the extension should be justified under the numerical conditions we have applied.

For a single-layered cylinder (i.e., $N=1$ ), the fourthorder polynomial of $\zeta$ given by (35) reads as

$$
\begin{aligned}
U= & \frac{\pi}{2} r^{3} k L \zeta^{2}+\frac{\pi}{16} r^{3} L E h \Gamma^{2}\left(8-12 \zeta+5 \zeta^{2}\right) \\
& +\frac{3 \pi}{512 r} L E h \zeta^{2}\left(64 h^{2}+81 r^{2} \zeta^{2}\right),
\end{aligned}
$$

where $r \equiv r_{1}$ and $\zeta \equiv \zeta_{1}$. The spring constant $k$ is that associated with springs illustrated in the left panel of Figure 9. 


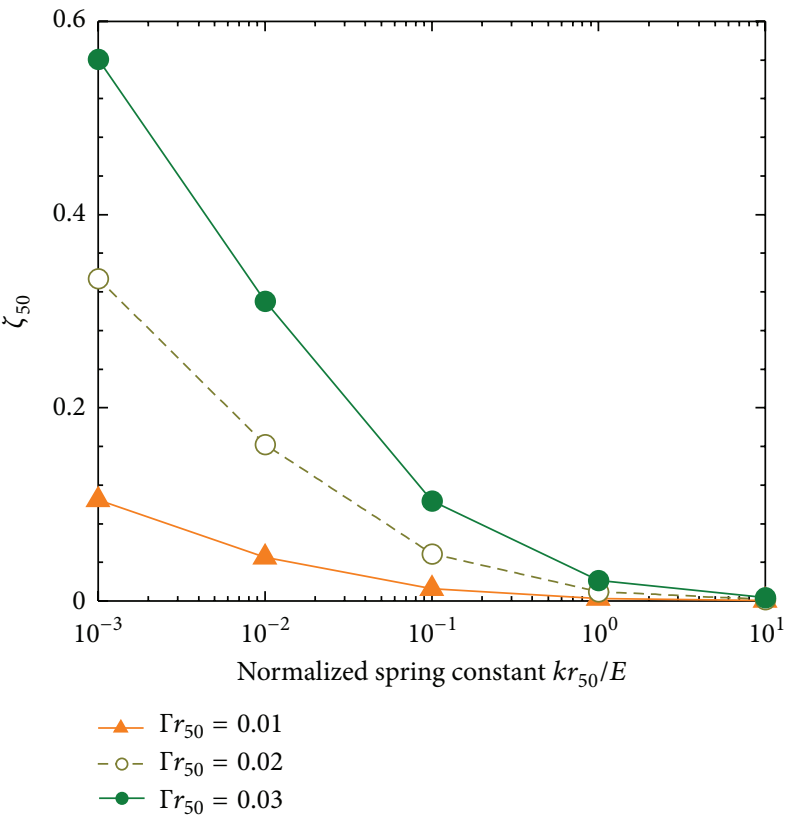

(a)

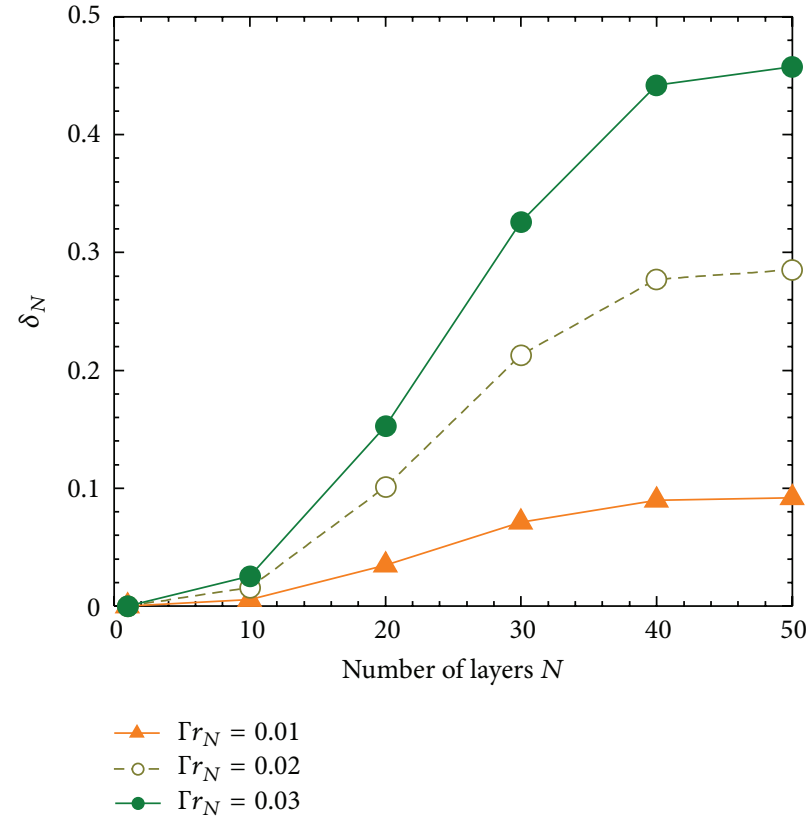

(b)

FiguRE 7: (a) Oblateness suppression caused by interlayer coupling in a 50-layered system. The coupling strength is quantified by the normalized spring constant $k r_{50} / E$ with $E$ being Young's modulus. (b) Difference in the short radius $r_{N} \zeta_{N}$ of deformed cross-sections between the weak and strong coupling conditions: $\delta_{N} \equiv\left(r_{N} \zeta_{N}\right)_{k r_{N} / E=0.001}-\left(r_{N} \zeta_{N}\right)_{k r_{N} / E=0.1}$.
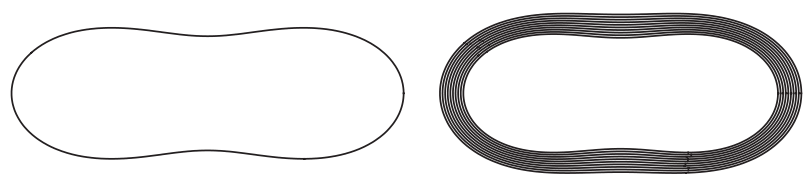

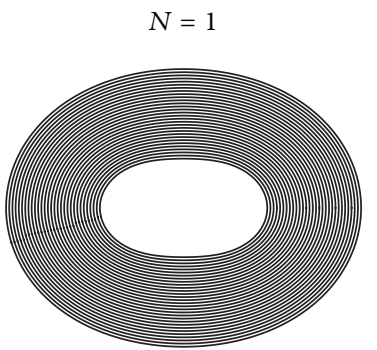

$N=30$

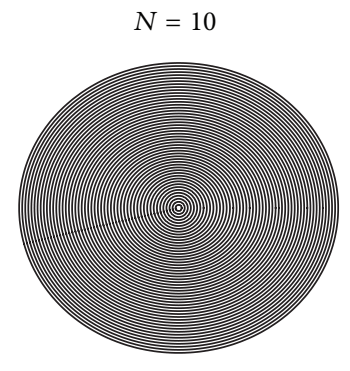

$N=50$
FiguRE 8: $N$-dependence of the cross-section. $\Gamma r_{N}=0.02$ and $k r_{N} / E=0.1$ are fixed for all images.

We can prove that the third-order equation, $\partial U / \partial \zeta=0$, with respect to $\zeta$ has a real solution and two imaginary ones as explained by the right panel in Figure 9. The real solution has the form of

$$
\zeta=\frac{1}{729(h / r)}\left[\sqrt[3]{p_{1} / 2}-p_{2} \sqrt[3]{2 / p_{1}}\right]
$$

with

$$
\begin{gathered}
p_{1}=q_{1}\left(\frac{h}{r}\right)^{3}(\Gamma r)^{2}+\sqrt{10^{16} q_{2}\left(\frac{h}{r}\right)^{6}(\Gamma r)^{4}+4 p_{2}^{3}}, \\
p_{2}=q_{3}\left(\frac{h}{r}\right)^{4}+q_{4}\left(\frac{h}{r}\right)^{2}(\Gamma r)^{2}+q_{5}\left(\frac{h}{r}\right) \frac{k r}{E}
\end{gathered}
$$

and $q_{1}=15306, q_{2}=2.3426, q_{3}=69984, q_{4}=58320, q_{5}=$ 93312 to five significant digits.

This $\Gamma$-dependence of $\zeta$ comes from our assumption of (32). Hence, the validity of our assumption can be tested by comparing with the exactly optimal oblateness of $\zeta_{u}=\zeta_{u}(\Gamma)$ and $\zeta_{v}=\zeta_{v}(\Gamma)$ that are defined by

$$
u=r \zeta_{u} \cos 2 \theta, \quad v=r \zeta_{v} \sin 2 \theta .
$$

Equation (A.6) is a general expression of displacements, free from our assumption, thus being accurate no matter if $\varepsilon_{\theta}$ vanishes or not. The optimal oblateness based on (A.6) is derived from $\partial U\left(\zeta_{u}, \zeta_{v}\right) / \partial \zeta_{u}=0$ and $\partial U\left(\zeta_{u}, \zeta_{v}\right) / \partial \zeta_{v}=0$, where $U\left(\zeta_{u}, \zeta_{v}\right)$ is a polynomial with respect to $\zeta_{u}$ and $\zeta_{v}$, having the function form different from $U(\zeta)$ given by (A.3).

Figure 10 shows the comparison between $\zeta$ and $\zeta_{u}$ for three different values of $k$. An almost equivalence between $\zeta(\Gamma)$ and $\zeta_{u}(\Gamma)$ has been confirmed for every $k$. Besides, $\zeta_{v}$ was found to be nearly equal to $-\zeta_{u} / 2$ at all $\Gamma$ and $k$ (not shown). From the data comparison, we conclude that the displacement expressions of (32) are effective for evaluating the oblateness of cylinders that exhibit nonvanishing $\varepsilon_{\theta}$. 


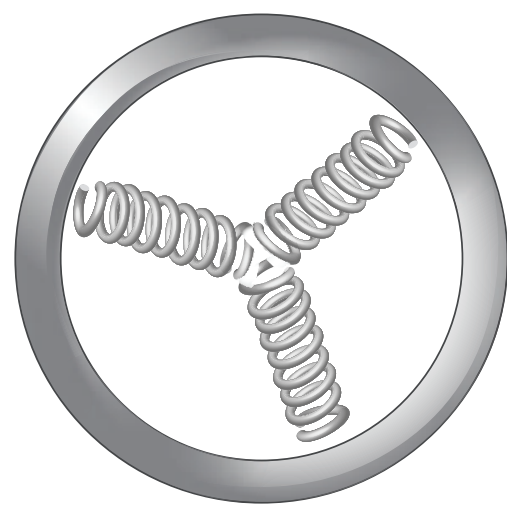

(a)

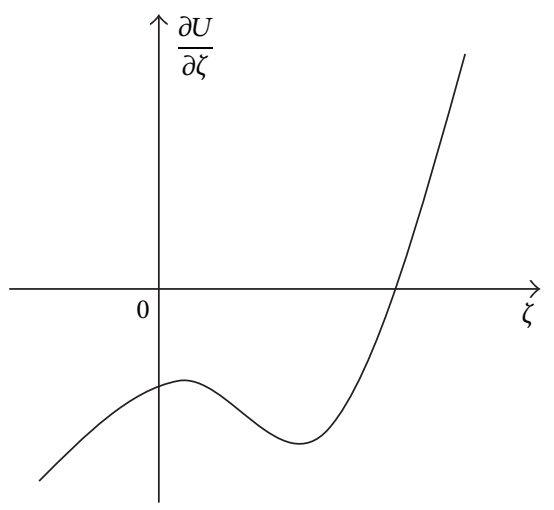

(b)

FIGURE 9: Left: sketch of the cross-section in a monolayered cylinder assumed. The effect of the coupling parameter $k$ is illustrated by virtual springs inside. Right: cubic curve of the $\zeta$-polynomial represented by $\partial U / \partial \zeta$.

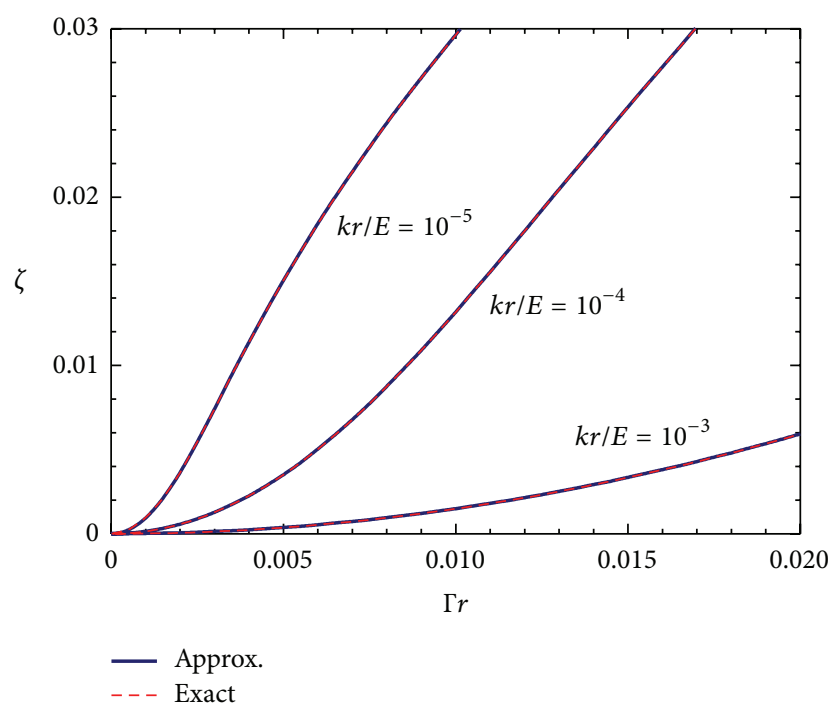

FIGURE 10: Data comparison between the approximated oblateness $\zeta$ and the exact one $\zeta_{u}$ for different values of $k$.

\section{Conflict of Interests}

The authors declare that there is no conflict of interests regarding the publication of this paper.

\section{Acknowledgment}

This work was supported by the University of Incheon, International Cooperative Research Grant, in 2013.

\section{References}

[1] H. Shima and M. Sato, Elastic and Plastic Deformation of Carbon Nanotoubes, Pan Stanford Publishing, Singapore, 2013.

[2] H. Shima, "Buckling of carbon nanotubes: a state of the art review," Materials, vol. 5, pp. 47-84, 2012.
[3] P. Gao, C. Zhan, and M. Liu, "Controlled synthesis of doubleand multiwall silver nanotubes with template organogel from a bolaamphiphile," Langmuir, vol. 22, no. 2, pp. 775-779, 2006.

[4] J. Zhu, J.-J. Li, and J.-W. Zhao, "Improve the refractive index sensitivity of coaxial-cable type gold nanostructure: the effect of dielectric polarization from the separate layer," Journal of Nanoparticle Research, vol. 15, article 1721, 2013.

[5] T. Delclos, C. Aimé, E. Pouget et al., "Individualized silica nanohelices and nanotubes: tuning inorganic nanostructures using lipidic self-assemblies," Nano Letters, vol. 8, no. 7, pp. 1929-1935, 2008

[6] C. Zhi, Y. Bando, C. Tang, and D. Golberg, "Boron nitride nanotubes," Materials Science and Engineering R: Reports, vol. 70, no. 3-6, pp. 92-111, 2010.

[7] M. Zheng, C. Ke, I.-T. Bae, C. Park, M. W. Smith, and K. Jordan, "Radial elasticity of multi-walled boron nitride nanotubes," Nanotechnology, vol. 23, no. 9, Article ID 095703, 2012.

[8] I. Kaplan-Ashiri, S. R. Cohen, K. Gartsman et al., "On the mechanical behavior of $\mathrm{WS}_{2}$ nanotubes under axial tension and compression," Proceedings of the National Academy of Sciences of the United States of America, vol. 103, no. 3, pp. 523-528, 2006.

[9] E. Kalfon-Cohen, O. Goldbart, R. Schreiber et al., "Radial compression studies of WS2 nanotubes in the elastic regime," Journal of Vacuum Science and Technology B: Microelectronics and Nanometer Structures, vol. 29, no. 2, Article ID 021009, 2011.

[10] J. Qiu, F. Zhuge, X. Li et al., "Coaxial multi-shelled $\mathrm{TiO}_{2}$ nanotube arrays for dye sensitized solar cells," Journal of Materials Chemistry, vol. 22, no. 8, pp. 3549-3554, 2012.

[11] D. Sept and F. C. MacKintosh, "Microtubule easticity: connecting all-atom simulations with continuum mechanics," Physical Review Letters, vol. 104, no. 1, Article ID 018101, 2010.

[12] Y. Zhao, L. An, and J. Fang, "Buckling instability of lipid tubules with multibilayer walls under local radial indentation," Physical Review E, vol. 80, no. 2, Article ID 021911, 2009.

[13] H.-S. Shen, "Nonlocal shear deformable shell model for bending buckling of microtubules embedded in an elastic medium," Physics Letters A, vol. 374, no. 39, pp. 4030-4039, 2010.

[14] M. Taj and J. Q. Zhang, "Analysis of vibrational behaviors of microtubules embedded within elastic medium by pasternak model," Biochemical and Biophysical Research Communications, vol. 424, no. 1, pp. 89-93, 2012. 
[15] Y. Gao and L. An, "A nonlocal elastic anisotropic shell model for microtubule buckling behaviors in cytoplasm," Physica E: Low-Dimensional Systems and Nanostructures, vol. 42, no. 9, pp. 2406-2415, 2010.

[16] M. Sato, M. H. Patel, and F. Trarieux, "Static displacement and elastic buckling characteristics of structural pipe-in-pipe crosssections," Structural Engineering and Mechanics, vol. 30, no. 3, pp. 263-278, 2008.

[17] T. Ozbakkaloglu, "Compressive behavior of concrete-filled FRP tube columns: assessment of critical column parameters," Engineering Structures, vol. 51, pp. 188-199, 2013.

[18] B. Başal and A. Ünal, "Numerical evaluation of a triple concentric-tube latent heat thermal energy storage," Solar Energy, vol. 92, pp. 196-205, 2013.

[19] H. Shima and M. Sato, "Multiple radial corrugations in multiwalled carbon nanotubes under pressure," Nanotechnology, vol. 19, no. 49, Article ID 495705, 2008.

[20] H. Shima, M. Sato, K. Iiboshi, S. Ghosh, and M. Arroyo, "Diverse corrugation pattern in radially shrinking carbon nanotubes," Physical Review B, vol. 82, no. 8, Article ID 085401, 2010.

[21] L. G. Brazier, "On flexure of thin cylindrical shells and other thin sections," Proceedings of the Royal Society of London A, vol. 116, no. 773, pp. 104-114, 1927.

[22] E. Reissner, "On finite pure bending of cylindrical tube," Österreichisches Ingenieur-Archiv, vol. 15, pp. 165-172, 1961.

[23] P. Seide and V. I. Weingarten, "On the buckling of circular cylindrical shells under pure bending," Journal of Applied Mechanics, vol. 28, no. 1, pp. 112-116, 1961.

[24] H. Shima, S. Ghosh, M. Arroyo, K. Iiboshi, and M. Sato, "Thinshell theory based analysis of radially pressurized multiwall carbon nanotubes," Computational Materials Science, vol. 52, no. 1, pp. 90-94, 2012.

[25] C. R. Calladine, Theory of Shell Structures, Cambridge University Press, Cambridge, UK, 1983. 

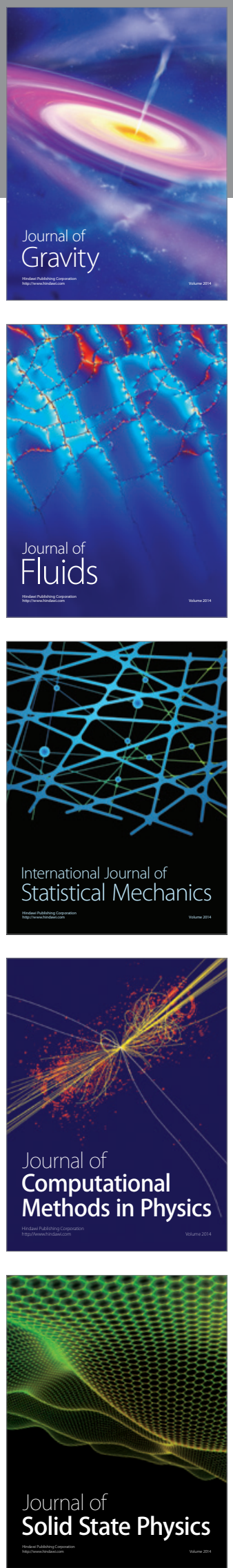

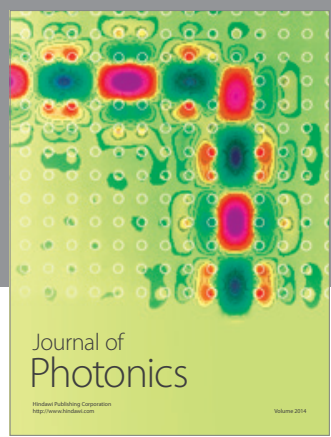

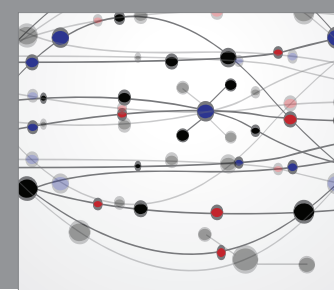

The Scientific World Journal

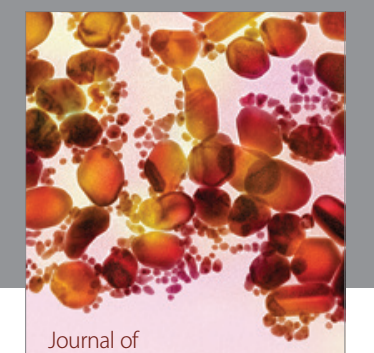

Soft Matter
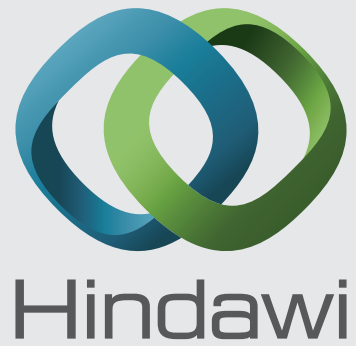

Submit your manuscripts at

http://www.hindawi.com
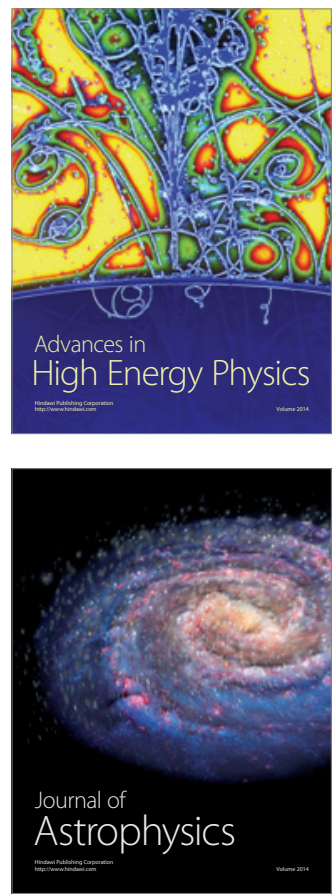
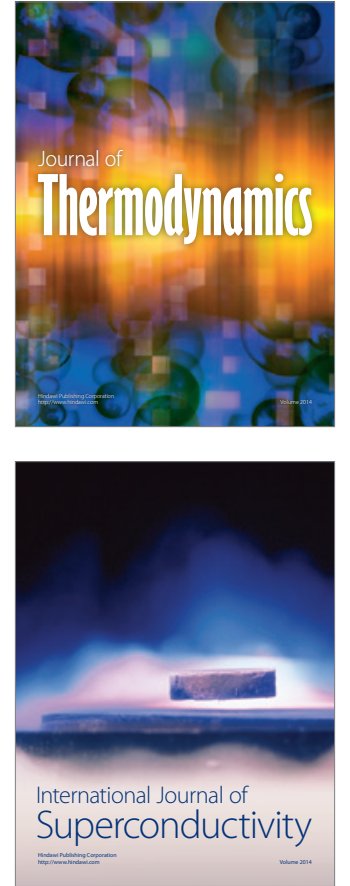
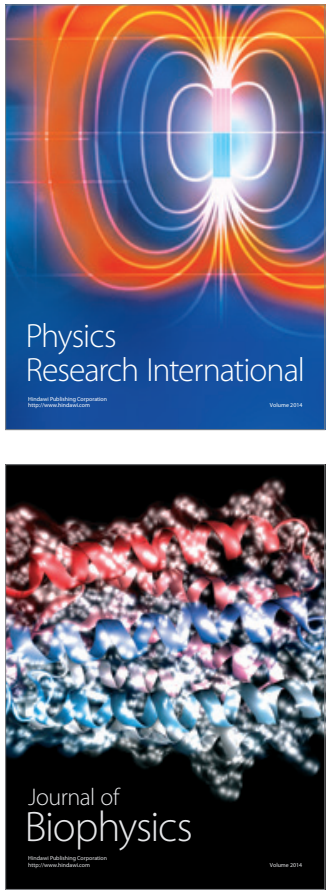
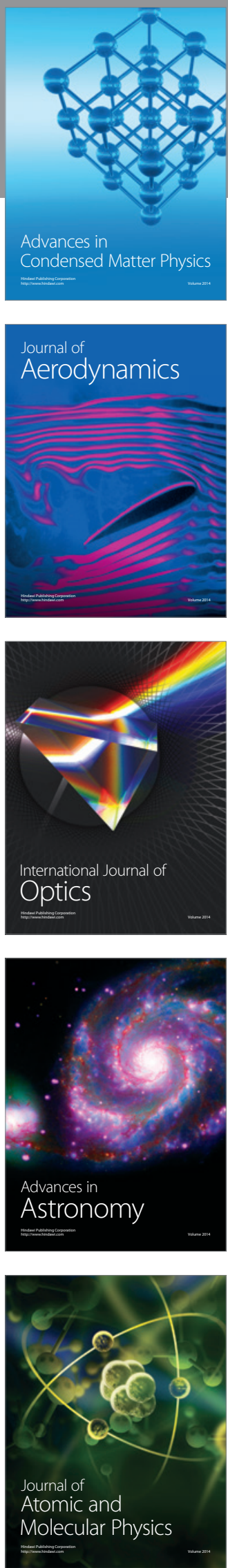